\title{
Finite Element Model Calibration Approach for Ares I-X
}

\author{
Lucas G. Horta ${ }^{1}$, Mercedes C. Reaves ${ }^{1}$, Ralph D. Buehrle ${ }^{2}$, Justin D. Templeton ${ }^{2}$, \\ Daniel R. Lazor ${ }^{3}$, James L. Gaspar ${ }^{4}$, Russel A. Parks ${ }^{3}$, and Paul A. Bartolotta ${ }^{5}$
}

\begin{abstract}
Ares I-X is a pathfinder vehicle concept under development by NASA to demonstrate a new class of launch vehicles. Although this vehicle is essentially a shell of what the Ares I vehicle will be, efforts are underway to model and calibrate the analytical models before its maiden flight. Work reported in this document will summarize the model calibration approach used including uncertainty quantification of vehicle responses and the use of nonconventional boundary conditions during component testing. Since finite element modeling is the primary modeling tool, the calibration process uses these models, often developed by different groups, to assess model deficiencies and to update parameters to reconcile test with predictions. Data for two major component tests and the flight vehicle are presented along with the calibration results. For calibration, sensitivity analysis is conducted using Analysis of Variance (ANOVA). To reduce the computational burden associated with ANOVA calculations, response surface models are used in lieu of computationally intensive finite element solutions. From the sensitivity studies, parameter importance is assessed as a function of frequency. In addition, the work presents an approach to evaluate the probability that a parameter set exists to reconcile test with analysis. Comparisons of pre-test predictions of frequency response uncertainty bounds with measured data, results from the variancebased sensitivity analysis, and results from component test models with calibrated boundary stiffness models are all presented.
\end{abstract}

\section{INTRODUCTION}

The process of model calibration involves reconciling differences between test and analysis. This process requires both heuristics and quantitative methods to assess model deficiencies, to evaluate parameter importance, and to compute required changes. Although model update (calibration) has been a very prolific area of research in the US and abroad, no single technique is universally accepted and at times, when viewed from a deterministic viewpoint, Avitabile in [1] referred to it as a problem with endless possibilities. For years, the commercially available tools to address the update problem relied on sensitivity information to judge the relative importance of parameters and to assist in making model changes. Friswell in [2] discusses many of the conventional sensitivity based approaches, some implemented in many commercial tools. These tools, in the hands of experienced engineers, provide heuristic approaches for model calibration that work very well in reconciling differences between test and analysis, but often provide unrealistic parameter changes and give little insight into the probabilistic nature of the problem. This aspect of the problem that has prompted extensive research with noteworthy contributions from Hasselman et al., [3]; Herendeen et al., [4]; Alvin, [5]; Farhat and Hemez, [6], aimed at addressing uncertainty. Hasselman discussed propagation of parameter uncertainty in frequency response calculations and presented various approaches to handle variability of response values near dynamic resonant conditions. Herendeen et al. [4] discussed a mathematical procedure using multi-disciplinary optimization to conduct analysis/test calibration studies of frequency response data. Alvin [5] extended a procedure developed by Farhat and Hemez, [6] to improve convergence and to incorporate uncertainty information into the estimation process, taking full advantage of the model structure and sensitivity. To their credit, very high dimensional problems have been calibrated successfully using these techniques. However, in the end the question about realism of updated parameters is still unanswered. To properly address this question one would need to exploit the work that Montgomery [7] has done in terms of design of experiments and Analysis of Variance (ANOVA) as a means to judge parameter adequacy. In the work of Uebelhart, [8] tools from modern designs of experiments are relied heavily for uncertainty quantification and parameter selection. Regardless of the parameter selection approach, engineering judgment will always play a key role, and these tools are available to guide the analyst.

In the computational fluid dynamics (CFD) area, work by Oberkampf [9] and Roach [10] is leading the verification and validation of mathematical models effort. In this area, techniques for systematic assessments of model

\footnotetext{
${ }^{1}$ NASA Langley Research Center, MS 230, Hampton, VA 23681

${ }^{2}$ NASA Langley Research Center, MS 424, Hampton, VA 23681

${ }^{3}$ NASA Marshall Space Flight Center, Huntsville, AL 35812

${ }^{4}$ NASA Langley Research Center, MS 434, Hampton, VA 23681

${ }^{5}$ NASA Glenn Research Center, MS 49-B, Cleveland, OH 44135
} 
adequacy and uncertainty quantification have been established along with standards, and a common language. Unfortunately, these efforts and the methods associated with them are just now slowly migrating to other disciplines; see, for example, Thacker [11]. The principles for model verification and validation set forth by the CFD community are applicable to many other disciplines but the metrics for assessment need to be modified. Using current terminology, the work presented here is primarily a model calibration effort.

Fundamental to the success of the model calibration effort is a clear understanding of the ability of a particular model to predict the observe behavior even when the observed behavior is uncertain. The approach proposed in this paper is focused primarily on model calibration using parameter uncertainty propagation and quantification, as opposed to a search for a reconciling solution. The process set forth follows a two-step approach. First, ANOVA is used for parameter sensitivity, which is followed by uncertainty propagation to evaluate uncertainty bounds and to gage the ability of the model to explain the observed behavior. Once this process is completed, optimization is used to find a reconciling solution. This approach was demonstrated by Horta [12].

Key to uncertainty propagation is the calculation of both single and multi-parameter variances. Because of the computational burden associated with variance-based sensitivity estimates, the approach in this report uses response surface models to estimate the frequency response functions (FRF) from which the variance is computed. Response surface modeling is based on the Extended Radial Basis Functions (ERBF) as described by Mullur [13-14].

To quantify the agreement between test and analysis for dynamic problems, it is common to use FRF data. Normally hundreds of sensors are placed on a structure to identify frequencies and mode shapes. If FRFs are used as calibration metrics, the number of metrics equals the product of the number of sensors times the number of inputs, which is often a large number. To reduce the number of metrics used for comparison, the Principal Values (PV) (also known as Principal Components (PC)) of the FRF are used instead. Incidentally, to expand this metric across multiple models, PV uncertainty bounds for the maximum of all maxima and the minimum of all minima are easily computed along with the probability of observing such values as a function of frequency, see Horta [15]. These bounds are then used to determine the probability that a solution exists that reconciles test results with analysis. To obtain a parameter set that reconciles the model with test, nonlinear optimization as described by Lewis [16] is used with a quadratic objective function to minimize the error between test and analysis. Hasselman [17] also used PC in a procedure he calls a PC-based statistical energy analysis, for generic uncertainty quantification and comparison of frequency responses. Our approach is very similar with the addition of PV maximum and minimum bounds. Future work needs to incorporate a probabilistic performance metric.

\section{DESCRIPTION OF ARES I-X VEHICLE}

The Ares I-X vehicle shown in figure 1, consists of the First Stage (FS), Upper Stage Simulator (USS), and the Crew Module and Launch Abort System (CM/LAS). This demonstration vehicle uses heritage shuttle first stage booster technology with newly designed components to mimic the mass loading of the Ares I vehicle. Because heritage hardware is used, and due to scheduling issues, only two components of the vehicle are tested prior to the flight vehicle modal test. Stack 5 (SS5), shown in figure 1, is the first major component tested. To minimize interference with the vehicle assembly schedule, SS5 is tested on a non-flight interface adapter while awaiting integration into the flight vehicle. This non-flight boundary interface adapter, facilitates access and transportation, but is not part of vehicle. Nonetheless, a finite element model (FEM) of the adapter is also part of the model evaluation.

Stack 1 (SS1) is the second major component tested and is shown in Fig. 1. It is comprised of the Interstage 2, Interstage 1, Frustum, Forward Skirt Extension, Forward Skirt, and $5^{\text {th }}$ Segment Simulator. This component is also tested while resting on a metal frame at the Vehicle Assembly Building (VAB) at the Kennedy Space Center. Because of the unconventional boundary conditions for both component tests, data analysis presented some unusual challenges.

Finally, a flight vehicle modal test is completed with the vehicle resting on hold-down posts on the Mobile Launcher Platform (MLP), shown in figure 2. This configuration is the closest to the flight configuration, and therefore, it is the most relevant configuration for guidance and navigation model verification.

\section{ARES I-X FINITE ELEMENT MODEL DESCRIPTION}

Figure 2 shows the Ares I-X flight vehicle FEM. The FS, USS, and CM/LAS finite element models were delivered by independent product teams (IPT). The three FEMs are connected with rigid elements (RBE2) at each interface. 
Between each of the three FEMs there are 24 connection points. These models reference a global coordinate system and have a unique numbering system to simplify the model integration effort. The Ares I-X modeling approach is consistent with current modeling practices for this type of structure. The vehicle skin is modeled using shell elements (CQUAD4 and CTRIA3), and section flanges and support bracing are modeled using beam elements. Lump masses such as nose tip, engines, splice plates, and umbilical are modeled using concentrated masses and are attached to the vehicle using rigid bar elements (RBE2). Constraint elements (RBE3) are used to attach platforms, secondary structures, and concentrated masses. Joints on the upper stage and first stage segments as well as ground and non-flight hardware boundary interface for the SS5 and SS1 test configurations are modeled with CBUSH spring elements.

\section{PROBLEM FORMULATION}

Model calibration begins with an initial assessment of the model adequacy for its intended use. For example, if the model is being developed to predict loads, detailed analysis of critically loaded regions must be emphasized. On the other hand, if the model is developed to support control design, critical structural modes must be measured and calibrated against analysis. The intended use determines the type of test and calibration process to follow. Metrics for the vehicle are established by the Guidance and Navigation Group in terms of allowable excursions from the nominal Ares I-X free-free model. Specifically, discrepancies in the frequency from test and analysis must be less than $10 \%$ for the $1^{\text {st }}$ bending pair and less than $20 \%$ for higher frequency modes; node locations for the $1^{\text {st }}$ three bending pairs must be within $+/-100$ inches of the nominal, and modal displacement differences must be less than $20 \%$ for the first bending pair and $50 \%$ for higher frequency modes. Although these metrics are for the free-free configuration, they are also used to evaluate the vehicle model on the MLP. The ability to observe the modes of interest of any modal test depends heavily on proper pre-test analysis, adequate sensor count, and sensor/shaker placement. Readers are referred to Buehrle [18] for details on the pre-test analysis and test configuration. Finally, the calibration process followed during test is shown in figure 3. Specific elements of the process are described next.

\subsection{Parameter Selection}

This step is perhaps the most difficult one because it requires first-hand knowledge of the assumptions and approximations used during model development. Also, because heritage hardware components are involved, those components and their parameters have not been considered for calibration; specifically, the first stage booster and the MLP. Collecting the necessary information for this step requires interviews with model developers and complete familiarization with all model elements.. After selecting an initial parameter set, the next step is the selection of parameter uncertainty models. These uncertainty models describe any a priori knowledge of the parameter variations in the form of distribution functions. In this problem, since there is no uncertainty information available, uniform distributions functions are used throughout the paper. As a consequence, all parameter updates resulting from this process are equally acceptable. Furthermore, parameter bounds are selected to assure that updates are acceptable from an engineering viewpoint.

The uncertainty propagation and quantification step requires executing the FEM multiple times with parameter variations as prescribed by the uncertainty model. This phase reveals outcomes that are highly probable, determines bounds for the response of interest, and is aimed at answering the question: what is the probability that the model can predict the measured data? Probability assessments are all based on discrete probabilities computed using a prescribed number of FEM runs. For example, using solutions obtained from $n$ different parameter sets, the discrete probability of observing a particular output is simply $1 / n$. Our goal in selecting a parameter set is to make the probability of capturing the measured response high. In other words, to find a parameter set that makes the analysis bounds as encompassing as possible.

Results from the parameter uncertainty study are used directly to create response surface models for use with a variance-based approach to determine parameter importance. Once the important parameters are identified, our ability to calibrate the model is assessed in terms of probability bounds. If the probability of finding a reconciling solution is greater than zero (i.e., test is within the uncertainty bounds), parameter updates are sought using the nonlinear optimization. Otherwise the parameter selection and bounds must be re-visited and modified.

\subsection{Analysis of Variance}

Parameter sensitivity in most engineering fields is often associated with derivative calculations at specific parameter values. However, for analysis of systems with uncertainties, sensitivity studies are often conducted using ANOVA. In classical ANOVA studies, data is collected from multiple experiments while varying all parameters (factors) and also while varying one parameter at a time. These results are then used to quantify the output response variance due to variations of a particular parameter as compared to the total output variance 
when varying all the parameters simultaneously. The ratio of these two variance contributions is a direct measure of the parameter importance.

Sobol in [19] and others [20-22] have studied the problem of global sensitivity analysis using variance measures. Sobol developed the "so called" Sobol indices to provide a measure of parameter sensitivity. In his formulation the variance is computed using the following expressions;

$$
\begin{aligned}
& \mu \cong \frac{1}{N} \sum_{i=1}^{N} \hat{y}\left(v_{i}\right) \\
& D+\mu^{2} \cong \frac{1}{N} \sum_{i=1}^{N} \hat{y}^{2}\left(v_{i}\right) \\
& D_{x}+\mu^{2} \cong \frac{1}{N} \sum_{i=1}^{N} \hat{y}\left(v_{i}\right) \hat{y}\left(x_{i}, z_{i}\right)
\end{aligned}
$$

where $\mu$ is the mean value of the response of interest $y, D$ is the total variance computed using $N$ samples, and $D_{\mathrm{x}}$ is the single parameter variance due to parameter $x$. Note that, $v_{i}$ refers to the $i^{\text {th }}$ sample of the parameter vector and $x_{i}$ is a parameter within $v_{i}$ about which the variance is being evaluated, whereas $z_{i}$ are all other parameters that comprise $v_{i}$ not including $x_{\mathrm{i}}$. To properly evaluate Eq. (1), one needs at least $2 N$ function evaluations; one where all variables are randomly sampled, and a second set where all but $x_{i}$ are re-sampled. With this information the $1^{\text {st }}$ Sobol index is computed as $S_{x}=D_{x} / D$. Of course, for problems with $m$ parameters, there are $m$ possible first order factors. Equation (1) is easily extended to study two or more parameter (factor) interactions, as described in [19], simply by adjusting the number of parameter that gets re-sampled. Only first factors are studied in this paper.

Depending on the type of structure being analyzed, FEM and their solutions can be computationally intensive. Because the parameter selection process relies on statistical analysis of the response data, it is important that no FEM solution is wasted. A way to capture information from every computed solution, as parameters are changed, is to use response surface models, i.e., surrogates. For this purpose, the Extended Radial Basis Function ERBF as developed by Mullur [13-14] is used. With this surrogate, it is now possible to conduct ANOVA in cases where computing thousands of FEM solutions directly is prohibitively expensive.

A final note on ANOVA using Sobol's approach regards convergence of the variance estimates. Although Ref. [21] discusses the asymptotic behavior of the variance estimates when using Eq. (1), for cases where a surrogate model is used instead of the FEM, the variance estimates are only as good as our surrogate model. Nonetheless, this approach provides an excellent way to rank variable importance when only a limited number of FEM solutions are possible.

\subsection{Goodness Metrics}

Metrics for dynamic problems are more appropriately provided in terms of mode shapes, frequencies, and FRF. One commonly used metric is the orthogonality criterion. This metric compares mode shapes extracted from measured FRF with FEM mode shapes weighted using the analytical mass matrix. Because this metric has been historically used, it is one of two metrics computed and reported. A second metric compares differences in the FRF from test and analysis directly. For optimization and uncertainty quantification studies this second metric is preferred and used for model calibration.

When studying the effect of parameter changes, Sobol is used to evaluate variances in the FRF at each frequency due to parameter variations. Instead of comparing each input/output pair, data is compared in terms of the Principal Values (PV) of the FRF. These PVs are computed as the singular value decomposition of the FRF matrix at each frequency. For cases with multiple singular values, only the maximum and minimum are compared.

\section{DISCUSSION OF RESULTS}

Results are presented for the component test first to make it easier for the reader to follow. Results for Stack 5 are presented first followed by Stack 1, and then the flight vehicle. 


\subsection{Stack 5 Model Calibration}

The first major component tested is the Command Module and Launch Abort System (SS5) as shown in figure 4. To facilitate access to the interior of the structure while on the ground, a service access structure (bottom yellow section) is mated and tested with the SS5 hardware, as shown in the photograph. Although this section is not part of the flight hardware, it is modeled, tested, and reported with the results. Figure 5 shows pre-test prediction of the $1^{\text {st }}$ three pairs of bending modes with their corresponding frequencies. Results from an independent traceability study by Tuttle [23] that considered subsystem coupling of SS5 with the flight vehicle concluded that although several modes of SS5 contribute to the total strain energy, the $1^{\text {st }}$ bending pair is the most important set and therefore should be the focus for this component test.

Analysis of Variance for SS5- is one of two tools used for selection of critical parameters. To begin this analysis, a second order ERBF response surface model with 8 parameters, as defined in Table 1, is initially created from 400 FEM eigenvalue solutions to provide modal frequencies and mode shapes as a function of boundary stiffness. From these 400 runs, the FRF is synthesized for displacements outputs and is used to evaluate FRF variations as a result of parameter variations. Initially, FEM runs are executed with parameter values uniformly distributed between the bounds shown in Table 1. By construction, the ERBF surrogate model matches each of the 400 solutions exactly. To study parameter contributions to the FRF variance, the ERBF surrogate is used instead of the FEM to generate hundreds (often thousands) of predictions and to compute the Sobol indices according to Eq. (1). Figure 6 shows the results from the Sobol calculation as a function of frequency with $N$ set to 1000 in equation 1. Colors are used to depict contribution of the individual parameter to the total variance where the total contribution from all 8 parameters is less than or equal to 1 . Frequency values with no colors are simply areas where the variance is not computed. As an example on how to use this information, suppose that changes to regions lower than $14 \mathrm{~Hz}$ are needed, then changes to parameters KV1 and KV3 (vertical stiffness of quadrant I and III) will produce the largest variation in the maximum principal value of the FRF. Note that quadrants, labeled in figure 6 using roman numerals I-IV, correspond to parameters labeled 1-4. For reference, at the top of figure 6 is a plot of the maximum PV as a function of frequency (with the nominal model) to indicate resonant frequencies near $4 \mathrm{~Hz}, 12 \mathrm{~Hz}$, and $26 \mathrm{~Hz}$. This sensitivity information is critical to understand how to properly set parameters before any optimization is performed. More importantly, it provides users with a per frequency map of parameter importance. Although individual parameters can dominate the variance in certain frequency ranges, variance alone does not provide information on the magnitude of the changes. To study magnitude variations, the approach using PV bounds is presented next.

SS5 Principal Value Uncertainty Analysis- The objective of this step is to assess variations in the FRF and to determine FRF bounds due to parameter variations. Using 400 parameters sets the maximum and minimum principal value across all models are computed and plotted as a function of frequency. These results are used not only as bounds for the measured FRF but also to estimate probabilities. Intuitively, if the response from 400 FEM models falls within the bounds then the probability of measuring a value outside the bounds must be less than $1 / 400$, if the parameter uncertainty model is adequate. Figure 7 a shows in solid-red the analysis bounds due to variations in the boundary stiffness computed prior to test, and the blue-dashed line shows the test data PV. Although the test data is from 58 accelerometers (with two shakers), the acceleration FRFs are scaled using frequency to compare results in terms of displacements. Incidentally, all analytical modes are assumed to have damping levels of $0.5 \%$. Two observations are in order regarding the results shown in figure $7 a: 1)$ variations in the PV values due to boundary stiffness changes are so small that the bounds looks just like the PV (see figure $8 a$ ), and 2) since our test data is outside the uncertainty bounds, the model representation is not adequate. The problem with this model is that the structure is on the ground without physical constraints, which makes the boundary stiffness unknown (epistemic uncertainty). It is clear that our initial estimate is incorrect and requires a second look.

Because of project time constraints, model calibration for SS5 concentrated exclusively on the boundary stiffness as the main source of discrepancies. After a post-test look at the model, it is determined that the boundary stiffness is two orders of magnitude lower than initially estimated. In addition, a discrepancy was found involving inertia properties used to simulate the engine mass. After adjusting the boundary stiffness uncertainty model as shown in Table 2 and correcting for the simulated engine inertias, results with the updated bounds are shown in figure $7 \mathrm{~b}$ along with the test data PV. With these corrections, the measured response is now within the analysis uncertainty bounds (for the $1^{\text {st }}$ two pair of modes) and parameter optimization can now proceed to determine stiffness parameters to reconcile test with analysis. Figure 8a shows the PV for the pre-test nominal model (solid- 
red) and test (dashed-blue); whereas figure $8 \mathrm{~b}$ shows results with the optimized boundary stiffness. Although results now are more in line with the measured FRF data, improvements are also seen in the orthogonality results.

Figure 9a shows orthogonality results comparing the pre-test FEM mode shapes (ordinate) with mode shapes identified from test (abscissa); whereas, figure $9 \mathrm{~b}$ shows results with the optimized boundary parameters. When examining these results, recall that orthogonality values range from 0 to 1 with black squares corresponding to values of 1 ; i.e. black squares indicate exact match. It is clear that updates to the boundary stiffness helped correct for errors in the $1^{\text {st }}$ pair of modes principal direction as well as reducing their frequency error. With this correction, the $1^{\text {st }}$ mode frequencies are within $2.9 \%$. Since the traceability study in [23] indicated that only the first bending mode pair is critical, no further updates are pursued.

Because models with uncertainty are being compared to test, test uncertainty is also critical. Unfortunately, in order to properly address this question one would need additional data, which for economic reasons have not been collected. Of the small number of data sets collected, PV values for test data is studied to determine if there is significant variability across different tests. With the limited number of tests, this variability, although not shown in the paper, is not significant enough to warrant additional tests.

\subsection{Stack 1 model calibration}

Stack1 is the second major component tested and is shown in figure 10. In this configuration, pre-test predictions of target modes are shown in figure 11 with the boundary modeled using springs to ground. As with SS5, results from an independent traceability study identified modes 1, 2, 6, 7 and 8 as our target modes for this component test.

SS1 Analysis of Variance- begins again by creating a second order ERBF response surface model with 8 parameters from 300 FEM eigenvalue solutions computed while varying the boundary stiffness. In this case the boundary is also divided into quadrants and stiffness parameters for each quadrant are varied independently. As before, the FRF response is synthesized for displacements outputs. To evaluate variations in the FRF as a result of parameter variations, FEM runs are executed with parameter values uniformly distributed between the bounds shown in Table 3. Variance results for Stack 1 are shown in figure 12 for each of the 8 boundary stiffness parameters. Again, only results for the variance of the maximum PV are shown; the nominal model PV is shown at the top of figure 12. In contrast to results for Stack 5 , for frequencies less than $5 \mathrm{~Hz}$, the lateral stiffness in all quadrants contribute significantly to the total variation.

SS1 Principal Value Uncertainty Analysis- For 300 parameter sets, the maximum and minimum PV across all FEM models are computed and plotted as a function of frequency. Figure 13a shows in solid-red the FEM PV bounds due to variations in the boundary stiffness computed prior to test and the blue-dashed line shows the test data PV. For this case, test data from 64 accelerometers (with 2 shakers) are used to recover the FRF and then converted to displacement FRF for comparison. It should be obvious that the measurements are outside the pretest model uncertainty bounds, and consequently the model is not adequate. As with SS5, the boundary stiffness is found to be two orders of magnitude softer than the initial estimate. After adjusting the uncertainty model for the boundary, the updated parameter ranges are defined in Table 4. With this updated set, the computed uncertainty bounds are now shown in figure 13b; solid-red is analysis and dashed blue is test. Damping levels for the model are again set to $0.5 \%$ for all modes. Since the boundary stiffness is unknown, it is selected in such a way as to ensure that the test data is captured within the bounds for the target modes. Figure 13b shows this assumption to be true; and, therefore, one can proceed to compute updated parameters using optimization.

For comparison, figure 14a shows the pre-test maximum and minimum PV with the nominal model in solid-red versus test in dashed-blue. Similarly, Figure 14b shows the results for SS1 with the optimized boundary parameters, shown in Table 4 . Note that PV matching of test with analysis is improved significantly for the $1^{\text {st }}$ mode pair.

Finally, orthogonality results for SS1 are shown in figure 15a using the pre-test model and $15 \mathrm{~b}$ uses the optimized boundary stiffness. Concentrating on the $1^{\text {st }}$ pair, the optimized values corrected a problem with the principal directions and also reduced the frequency errors. Although high frequency modes are difficult to observe with the limited instrumentation suite, qualitative assessments of test modes with orthogonality values greater than 0.8 
revealed that many of these modes resemble modes found in the analysis. No further updates are performed due to time constraints and the need to prepare for the flight vehicle test.

\subsection{Flight Test Vehicle (FTV) Model Calibration}

The flight vehicle calibration process follows the same steps as those described with the component tests. Since the Ares I-X vehicle is tested while mounted on four hold-down posts on the MLP, as opposed to free-free, target modes in this configuration are the $1^{\text {st }}$ four bending mode pairs. Figure 16 shows only the first mode of each pair. It is assumed that after calibrating the analytical model of the vehicle on the MLP, the MLP can be removed analytically to predict the free-free configuration. In addition, damping values obtained from this test can be allocated to the free-free modes.

FTV Analysis of Variance- Unlike the SS1 and SS5 component tests, the FTV boundary condition is relatively well defined. Consequently, parameters selected for uncertainty analysis and their variations must be supported with data collected from various sources. After a careful study of potential sources of uncertainty, the parameters defined in Table 5 are selected. The seven parameters used in this study included the USS joint stiffness, FTV/MLP interface stiffness, forward skirt Young modulus XL_SK_E, the CM/LAS Young modulus LAS_E, as well as ballast mass densities B_US_1/B_US_7. Ranges used are based on collected information regarding modeling assumptions and uncertainty in the information collected.

Figure 17 shows results for the variance analysis with the seven parameters. Note that the variance for the first two modes (about $0.2 \mathrm{~Hz}$ ) is dominated by ballast densities, whereas modes between 3 to $4 \mathrm{~Hz}$ are dominated by the USS joint stiffness.

FTV Principal Value Uncertainty Analysis- Due to time constraints, only 200 FEM runs are used to compute the PV bounds. Figure 18 shows results for the FEM analysis uncertainty bounds in solid-red and test in dashed blue. Two aspects of these results should be highlighted; 1) variations in the PV due to parameter variations are relatively small, and 2) the uncertainty bounds captures relatively well the measured data with only a few exceptions. Damping for the analysis is again assumed to be $0.5 \%$ for all flexible modes. Figure 19 shows the PV using the pre-test nominal model (solid-red) as compared to the measured values (dashed-blue). Again the agreement is good.

A final check examines orthogonality results of the pre-test nominal model with the identified modes from test. Results for this metric are shown in figure 20. Along the abscissa are test mode frequencies and along the ordinate are analysis frequencies. Modes circled correspond to target modes that need to be verified for controls. Frequency errors for those modes are under $6.5 \%$. Table 6 shows a summary of the identified frequencies and measured damping ranges (in brackets) using only random excitation data.

With this information and the requirements initially provided by the guidance and navigation team, the nominal Ares I-X model is found to be adequate for evaluation of the flight control system without need for additional parameters adjustments. Although there are certain areas where the model can be improved, these areas should not impact guidance performance of the vehicle based on the initial requirements.

\section{CONCLUDING REMARKS}

A procedure to conduct model calibration for the Ares I-X vehicle has been presented The approach uses a variance-based approach for parameter selection, nonlinear optimization to minimize the error between test and analysis, and multiple FEM models to bound the system response and to assess the probability of finding a reconciling solution. To alleviate the computational expense in performing variance-based sensitivity analysis, the approach uses a surrogate model to predict changes in the frequency response functions as parameters are varied. Uncertainty in the parameters and their effect on the frequency response function is studied in terms of Principal Values. Uncertainty bounds of the principal values are established across multiple models to allow one to determine the probability of finding a solution that reconciles test with analysis results. Model adequacy is easily ascertained by comparing the measured responses against PV uncertainty bounds.

Because of the use of unconventional boundary conditions during Stack 5 and Stack1 tests, the model calibration effort concentrated on boundary stiffness uncertainty characterization. In both cases, the initial boundary stiffness estimates were two orders of magnitude lower than initially thought. After adjusting the uncertainty models for the 
boundary stiffness to properly capture the measured data, optimization is used to find a set of parameters that reconciles test with analysis. This optimized parameter set is found to minimize the error between the test and analysis FRF. Because uncertainty data is not available, it is impossible to make probabilistic statements regarding confidence in the optimized boundary stiffness values. Although, the boundary stiffness parameters do not alter vehicle parameters directly, agreement of test with analysis for the target modes improved significantly after boundary adjustments. Likewise, our confidence in the nominal model is also improved.

Finally, results for the Ares I-X vehicle are presented and compared to predictions using the nominal model with uncertainty bounds computed prior to test. These results show that the nominal model is remarkably close to the pre-test predictions and complies with model verification metrics provided by the guidance and navigation group. For this reason, changes to the nominal model are not recommended.

\section{$7 \quad$ ACKNOWLEDGEMENTS}

Special thanks to the FEM development group for their continued support: Winifred Feldhaus (LaRC,FTV), Genevieve Dixon (LaRC,CM/LAS), Troy Mann (LaRC,CM/LAS), Richard Pappa (LaRC,CMILAS), Nickolas A. Vitullo (ATK, weight), Andrew Panetta (LaRC,weight/cg), William K. Thompson (GRC, upper stage), Jeremy Redden (ATK,first stage), Carl Pray (ATA, uncertainty analysis, Jones Trevor (GRC, upper stage), Jennifer Lange (GRC,upper stage). Thanks to our independent verification team of Jeff Lollock, Ryan Tuttle, and Joshua Hwung from Aerospace Corporation. Finally thanks to the KSC team; Russ Brucker, Trip Healey, Frank Walker, Stephanie Heffernan, Teresa Kinney, Todd Reeves, Kara Schmitt, Mark Tillett, and Carl Meyer.

\section{REFERENCES}

1. Avitabile, P.: "Model Updating- Endless Possibilities." Proceedings of the $18^{\text {th }}$ International Modal Analysis Conference, San Antonio, TX, Feb. 2000, pp. 562-570.

2. Friswell, M.I., and Mottershead, J.E.: Finite Element Model Updating in Structural Dynamics, Kluwer Academic Press, Dordrecht, 1995.

3. Hasselman, T.K., Chrostowski, J.D., and Ross, T.J.: "Propagation of Modeling Uncertainty Through Structural Dynamic Models," Proceedings of the $35^{\text {th }}$ AIAA/ ASME/ASCE/AHS/ASC Structures, Structural Dynamics, and Materials Conference, 1994, Paper No. AIAA 94-1316, SC.

4. Herendeen, D.L., Woo, L., Hasselman, T.K., and Zimmerman, D.C.: "Analysis-Test Correlation and Model Updating of Dynamic Systems Using MDO Software Tools," Proceedings of the $7^{\text {th }}$ AIAA/USAF/NASA/SSMO Symposium on Multidisciplinary Analysis and Optimization, 1998, Paper No. 98-4730, St. Louis, MO.

5. Alvin, K.F.: "Finite Element Model Update via Bayesian Estimation and Minimization of Weighted Residuals," AIAA Journal, Vol. 35, No. 5, 1997.

6. Farhat, C. and Hemez, F.M.: "Updating Finite Element Dynamic Models Using an Element-by-Element Sensitivity Methodology," AIAA Journal, Vol. 31, No. 9, 1993, pp. 1702-1711.

7. Montgomery D.C., Design and Analysis of Experiments, John Wiley \& Sons, New York, $5^{\text {th }}$ ed. 2001

8. Uebelhart, S.A., Miller, D.W., and Blaurock, C.: "Uncertainty Characterization in Integrated Modeling." Proceedings of the $46^{\text {th }}$ AIAA/ASME/ASCE/AHS/ASC Structures, Structural Dynamics, \& Materials Conference, April 2005, Austin TX., AIAA 2005-2142.

9. Oberkampf, W.L., Trucano, T.G., and Hirsch, C.: "Verification, Validation, and Predictive Capability in Computational Engineering and Physics." SAND 2003-3769, Feb. 2003.

10. Roach, P.P., Verification and Validation in Computational Science and Engineering, Hermosa Publishers, Albuquerque, NM, 1998.

11. Thacker, B.H.: "The Role of Non-determinism in Computational Model Verification and Validation." Proceedings of the $46^{\text {th }}$ AIAA/ASME/ASCE/AHS/ASC Structures, Structural Dynamics, \& Materials Conference, April 2005, Austin TX, AIAA 2005-1902.

12. Horta, L.G., Reaves, M.C., and Lew, J.-S.: "A Procedure for Static and Dynamic Model Update of Finite Element Models: Application to an Inflated/Rigidized Torus." Proceedings of the IMAC XXIV: Conference and Exposition on Structural Dynamics, 2006, St. Louis Missouri.

13. Mullur, A. and Messac, A.: "Extended Radial Basis Functions: More Flexible and Effective Metamodeling." AIAA Journal, Vol., 43, No. 6, June 2005.

14. Mullur, A. and Messac, A.: "Metamodeling Using Extended Radial Basis Functions: A Comparative Approach." Engineering with Computers (2006) 21: 203-217 DOI 10.1007/s00366-0005-7. 
15. Horta, L.G., Kenny, S.P., Crespo, L.G., and Elliott, K.B.: "NASA Langley's Approach to the Sandia's Structural Dynamics Challenge Problem." Computer Methods in Applied Mechanics and Engineering, Vol. 197, Issues 29-32, May 2008, also available at www.sciencedirect.com.

16. Lewis. R.W., Shepherd A., and Torczon, V.: "Implementing Generating Set Search Methods for Linearly Constrained Minimization." SIAM Journal on Scientific Computing, Vol. 29, No. 6, pp 2507-2530.

17. Hasselman T., Yap K., Yan H. and Parret, A.: "Statistical Energy Analysis by Principal Components for MidFrequency Analysis." Proceedings of the $43^{\text {rd }}$ AIAA/ASME/ASCE/AHS/ASC Structures, Structural Dynamics, and Materials Conference, April 2002, Denver, CO. AIAA 2002-1395.

18. Buehrle, R.D., Templeton, J.D., Reaves, M.C., Horta, L.G., Gaspar, J.L, Bartolotta, P.A., Parks, R.A., and Lazor, D.R,: "Ares I-X Launch Vehicle Modal Test Overview." Proceedings of IMAC XXVIII, Jacksonville, FL., February 1-4, 2010.

19. Sobol, I.M., Tarantola, S., Gatelli, D., Kucherenko, S.S, and Mauntz, W.: "Estimating Approximation Error When Fixing Unessential Factors in Global Sensitivity Analysis," Reliability Engineering and System Safety 92 (2007) 957-960, @ 2006 Elsevier LTD.

20. Mullershon, H., and Liebsher, M.: "Statistics and Non-Linear Sensitivity Analysis with LS-OPT and DSPEX." $10^{\text {th }}$ International LS-DYNA Users Conference, pp. 4-1,4-13.

21. Homma, T, and Saltelli, A.: "Importance Measures in Global Sensitivity Analysis of Nonlinear Models." Reliability Engineering and System Safety 52 (1996) 1-17, () 1996 Elsevier LTD.

22. Sudret, B.: "Global Sensitivity Analysis Using Polynomial Chaos Expansion," Reliability Engineering and System Safety 93 (2008) 964-979, @ 2007 Elsevier LTD.

23. Tuttle, R., Lollock, J.A, and Hwung, J.S.: "Identifying Goals for Ares I-X Modal Testing," Proceedings of IMAC XXVIII, Jacksonville, FL., February 1-4, 2010.

Table 1 Stack 5 Pre-test parameter definition

\begin{tabular}{|c|c|c|c|c|}
\hline No. & Parameter ID & $\begin{array}{l}\text { Lower } \\
\text { Bound }\end{array}$ & Nominal & $\begin{array}{l}\text { Upper } \\
\text { Bound }\end{array}$ \\
\hline 1 & Kv1 (Ibs//in) & $4.84 \mathrm{E}+07$ & $6.06 \mathrm{E}+07$ & $6.06 E+07$ \\
\hline 2 & $\mathrm{KI} 1$ (Ibs//in) & $4.84 \mathrm{E}+07$ & $6.06 \mathrm{E}+07$ & $6.06 \mathrm{E}+07$ \\
\hline 3 & Kv2 (Ibs//in) & $4.84 \mathrm{E}+07$ & $6.06 \mathrm{E}+07$ & $6.06 E+07$ \\
\hline 4 & KI2 (lbs//in) & $4.84 \mathrm{E}+07$ & $6.06 \mathrm{E}+07$ & $6.06 \mathrm{E}+07$ \\
\hline 5 & Kv3 (Ibs//in) & $4.84 \mathrm{E}+07$ & $6.06 \mathrm{E}+07$ & $6.06 E+07$ \\
\hline 6 & KI3 (Ibs//in) & $4.84 \mathrm{E}+07$ & $6.06 \mathrm{E}+07$ & $6.06 \mathrm{E}+07$ \\
\hline 7 & Kv4 (Ibs//in) & $4.84 \mathrm{E}+07$ & $6.06 \mathrm{E}+07$ & $6.06 \mathrm{E}+07$ \\
\hline 8 & $\mathrm{Kl} 4$ (Ibs//in) & $4.84 \mathrm{E}+07$ & $6.06 \mathrm{E}+07$ & $6.06 \mathrm{E}+07$ \\
\hline
\end{tabular}

Table 2 Stack 5 Post-test parameter definition and optimized solution

\begin{tabular}{|c|c|c|c|c|c|}
\hline No. & Parameter ID & Lower Bound & Nominal & Upper Bound & $\begin{array}{l}\text { Optimized } \\
\text { Solution }\end{array}$ \\
\hline 1 & Kv1 (lbs//in) & $5.45 E+05$ & $6.06 E+05$ & $3.33 E+06$ & $5.45 \mathrm{E}+05$ \\
\hline 2 & KI1 (Ibs//in) & $5.45 E+05$ & $6.06 E+05$ & $3.33 E+06$ & $6.01 \mathrm{E}+05$ \\
\hline 3 & Kv2 (lbs//in) & $5.45 E+05$ & $6.06 E+05$ & $3.33 E+06$ & $3.33 E+06$ \\
\hline 4 & $\mathrm{KI} 2$ (lbs//in) & $5.45 E+05$ & $6.06 E+05$ & $3.33 E+06$ & $1.09 E+06$ \\
\hline 5 & Kv3 (lbs//in) & $5.45 \mathrm{E}+05$ & $6.06 E+05$ & $3.33 E+06$ & $5.47 E+05$ \\
\hline 6 & KI3 (Ibs//in) & $5.45 E+05$ & $6.06 \mathrm{E}+05$ & $3.33 E+06$ & $5.94 \mathrm{E}+05$ \\
\hline 7 & Kv4 (lbs//lin) & $5.45 \mathrm{E}+05$ & $6.06 \mathrm{E}+05$ & $3.33 E+06$ & $3.33 E+06$ \\
\hline 8 & $\mathrm{KI}$ (Ibs//in) & $5.45 E+05$ & $6.06 \mathrm{E}+05$ & $3.33 E+06$ & $3.33 E+06$ \\
\hline
\end{tabular}


Table 3 Stack 1 pre-test parameter definition

\begin{tabular}{clrrr}
\hline No. & Parameter ID & Lower Bound & Nominal & Upper Bound \\
\hline 1 & KQ2RTh (Ibs/in) & $1.00 \mathrm{E}+06$ & $1.00 \mathrm{E}+07$ & $1.00 \mathrm{E}+08$ \\
2 & KVQ2 (Ibs/in) & $1.00 \mathrm{E}+06$ & $1.00 \mathrm{E}+07$ & $1.00 \mathrm{E}+08$ \\
3 & KQ3RTh (Ibs/in) & $1.00 \mathrm{E}+06$ & $1.00 \mathrm{E}+07$ & $1.00 \mathrm{E}+08$ \\
4 & KVQ3 (Ibs/in) & $1.00 \mathrm{E}+06$ & $1.00 \mathrm{E}+07$ & $1.00 \mathrm{E}+08$ \\
5 & KQ4RTh (Ibs/in) & $1.00 \mathrm{E}+06$ & $1.00 \mathrm{E}+07$ & $1.00 \mathrm{E}+08$ \\
6 & KVQ4 (Ibs/in) & $1.00 \mathrm{E}+06$ & $1.00 \mathrm{E}+07$ & $1.00 \mathrm{E}+08$ \\
7 & KQ1RTh (Ibs/in) & $1.00 \mathrm{E}+06$ & $1.00 \mathrm{E}+07$ & $1.00 \mathrm{E}+08$ \\
\hline 8 & KVQ1 (Ibs/in) & $1.00 \mathrm{E}+06$ & $1.00 \mathrm{E}+07$ & $1.00 \mathrm{E}+08$ \\
\hline
\end{tabular}

Table 4 Stack 1 Post-test parameter definition and optimized solution

\begin{tabular}{clcccc}
\hline No. & $\begin{array}{c}\text { Parameter } \\
\text { ID }\end{array}$ & $\begin{array}{c}\text { Lower } \\
\text { Bound }\end{array}$ & Nominal & $\begin{array}{c}\text { Upper } \\
\text { Bound }\end{array}$ & $\begin{array}{c}\text { Optimized } \\
\text { Solution }\end{array}$ \\
\hline 1 & KQ2RTh (Ibs/in) & $2.50 \mathrm{E}+05$ & $3.75 \mathrm{E}+05$ & $1.00 \mathrm{E}+06$ & $1.00 \mathrm{E}+06$ \\
2 & KVQ2 (lbs/in) & $2.50 \mathrm{E}+05$ & $3.75 \mathrm{E}+05$ & $1.00 \mathrm{E}+06$ & $8.03 \mathrm{E}+05$ \\
3 & KQ3RTh (Ibs/in) & $2.50 \mathrm{E}+05$ & $3.75 \mathrm{E}+05$ & $1.00 \mathrm{E}+06$ & $1.00 \mathrm{E}+06$ \\
\hline 4 & KVQ3 (Ibs/in) & $2.50 \mathrm{E}+05$ & $3.75 \mathrm{E}+05$ & $1.00 \mathrm{E}+06$ & $2.50 \mathrm{E}+05$ \\
5 & KQ4RTh (Ibs/in) & $2.50 \mathrm{E}+05$ & $3.75 \mathrm{E}+05$ & $1.00 \mathrm{E}+06$ & $1.00 \mathrm{E}+06$ \\
6 & KVQ4 (Ibs/in) & $2.50 \mathrm{E}+05$ & $3.75 \mathrm{E}+05$ & $1.00 \mathrm{E}+06$ & $6.32 \mathrm{E}+05$ \\
7 & KQ1RTh (lbs/in) & $2.50 \mathrm{E}+05$ & $3.75 \mathrm{E}+05$ & $1.00 \mathrm{E}+06$ & $1.00 \mathrm{E}+06$ \\
\hline 8 & KVQ1 (lbs/in) & $2.50 \mathrm{E}+05$ & $3.75 \mathrm{E}+05$ & $1.00 \mathrm{E}+06$ & $4.27 \mathrm{E}+05$ \\
\hline
\end{tabular}

Table 5 Flight vehicle pre-test parameter ranges

\begin{tabular}{|c|c|c|c|c|}
\hline No. & $\begin{array}{c}\text { Parameter } \\
\text { ID }\end{array}$ & $\begin{array}{l}\text { Lower } \\
\text { Bound }\end{array}$ & Nominal & $\begin{array}{l}\text { Upper } \\
\text { Bound }\end{array}$ \\
\hline 1 & USS_J (Ibs/in) & $4.84 \mathrm{E}+06$ & $1.00 \mathrm{E}+07$ & $2.63 E+07$ \\
\hline 2 & FTV/MLP_V (Ibs/in) & $2.43 E+08$ & $2.70 \mathrm{E}+08$ & 2.97E+08 \\
\hline 3 & FTV/MLP_L (Ibs/in) & $2.43 E+08$ & $2.70 E+08$ & 2.97E+08 \\
\hline 4 & B_US_1 (Ibs-s $\left.{ }^{2} / \mathrm{in}^{4}\right)$ & $6.44 \mathrm{E}-04$ & 7.32E-04 & 8.27E-04 \\
\hline 5 & B_US_7 (Ibs-s $\left.{ }^{2} / \mathrm{in}^{4}\right)$ & 5.63E-04 & 7.32E-04 & $8.20 \mathrm{E}-04$ \\
\hline 6 & X'̄_Fw'- dSkirt_E (Ibs/in $\left.{ }^{2}\right)$ & $2.70 \mathrm{E}+07$ & $3.00 \mathrm{E}+07$ & $3.30 \mathrm{E}+07$ \\
\hline 7 & LAS_E (Ibs/in $\left.{ }^{2}\right)$ & $8.91 \mathrm{E}+06$ & $9.90 \mathrm{E}+06$ & $1.09 E+07$ \\
\hline
\end{tabular}


Table 6 Summary of flight vehicle identified frequencies and damping

\begin{tabular}{cccl}
\hline Mode & Freq. (Hz) & Damp. (\%) & \\
\hline 1 & 0.18 & {$[0.48,0.83]$} & Y-X 1st bend \\
\hline 2 & 0.21 & {$[0.36,0.44]$} & Z-X 1st bend \\
3 & 1.06 & {$[0.28,0.35]$} & Y-X 2nd bend \\
\hline 4 & 1.19 & {$[0.39,0.43]$} & Z-X 2nd bend \\
\hline 5 & 1.87 & {$[2.22,2.95]$} & Z-X bend \&MLP \\
\hline 6 & 2.07 & {$[1.28,1.43]$} & Y-X bend \& MLP \\
7 & 2.91 & {$[0.96,1.40]$} & 3rd bend \% MLP \\
\hline 8 & 3.46 & {$[0.43,0.52]$} & 3rd Y-X bend \\
9 & 3.64 & {$[0.37,0.41]$} & 3rd Z-X bend \\
\hline 10 & 3.68 & {$[0.38,0.41]$} & Torsion \\
\hline 11 & 4.61 & {$[0.17,0.18]$} & 4th bend (45deg) \\
\hline 12 & 4.78 & {$[0.23,0.25]$} & 4th bend X-Y \\
13 & 6.17 & {$[1.35,1.38]$} & system \\
\hline 14 & 6.41 & {$[0.66,0.69]$} & system \\
\hline 15 & 6.66 & {$[1.29,1.40]$} & system \\
\hline
\end{tabular}
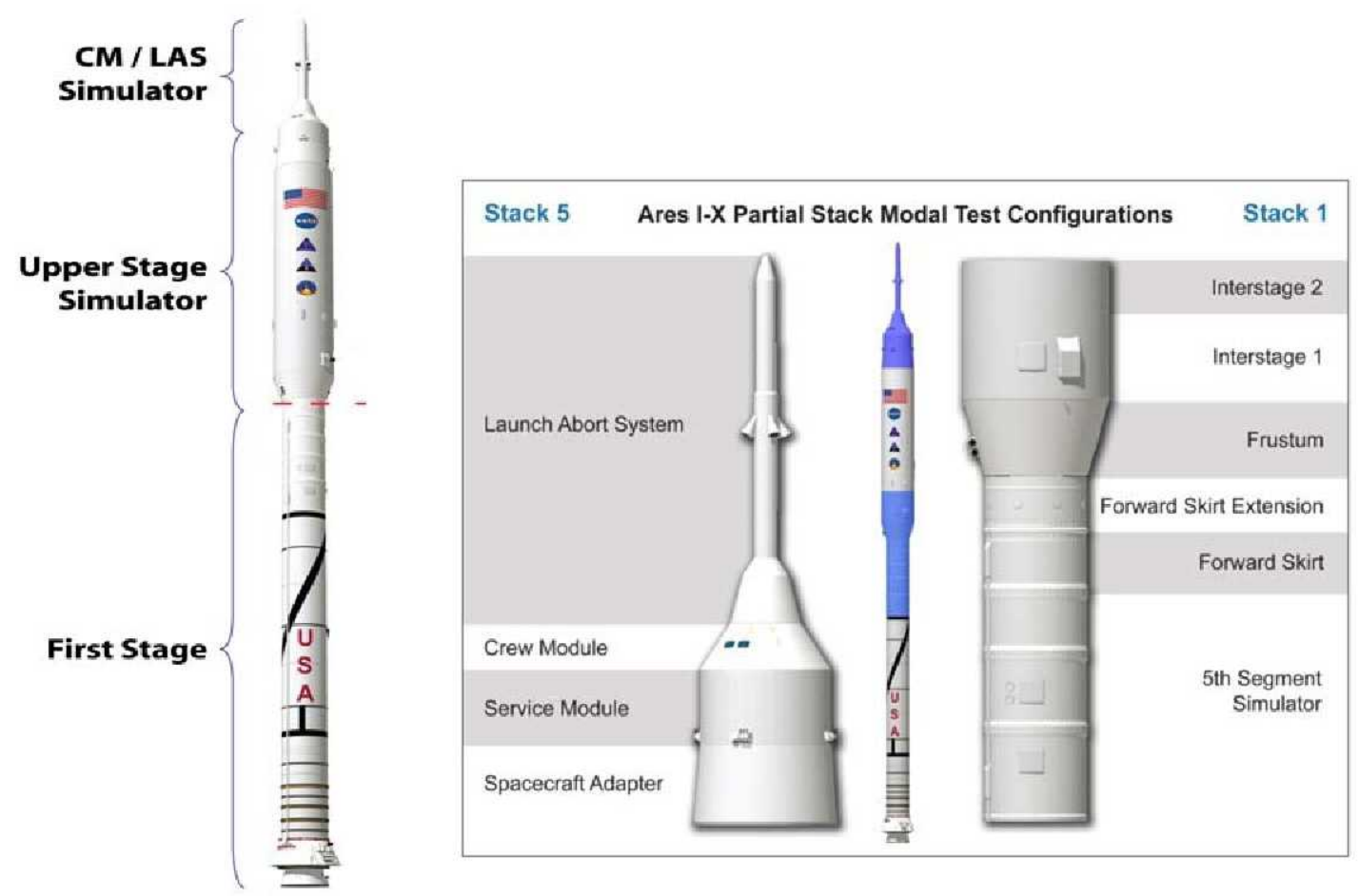

Figure 1 Ares I-X vehicle schematic and subcomponents tested 

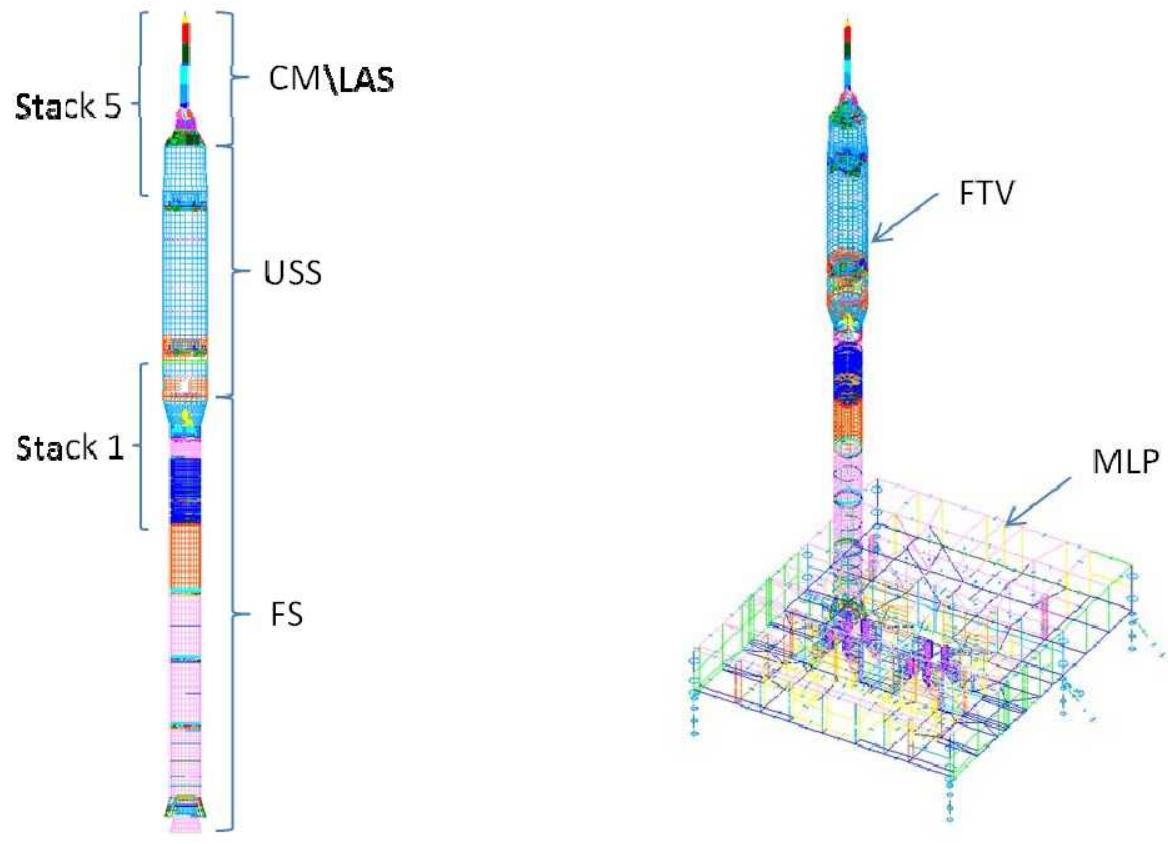

Figure 2 Finite element model schematic of the flight vehicle

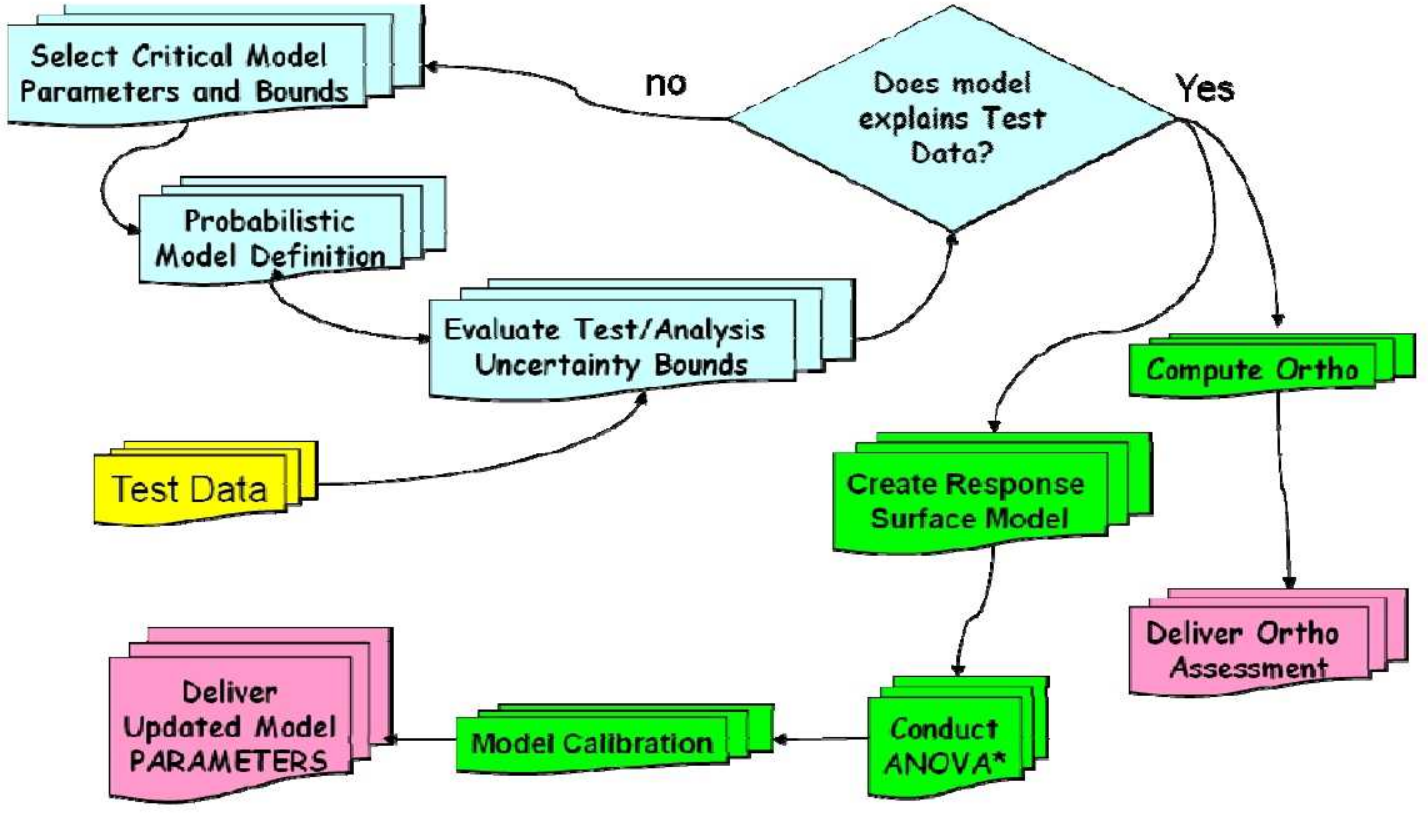

Figure 3 Model calibration process 

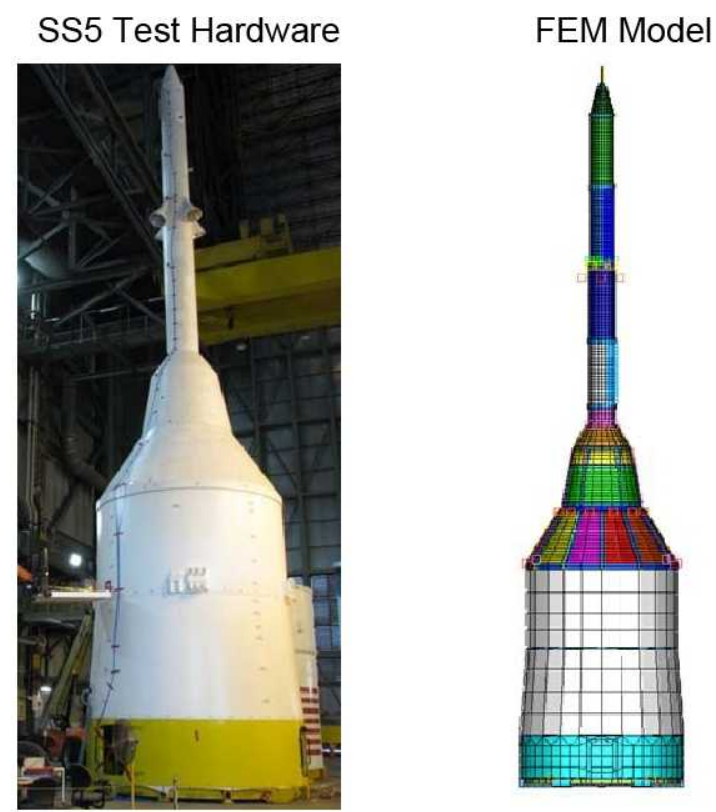

Figure 4 Ares I-X Command Module and Launch Abort System (CM-LAS)
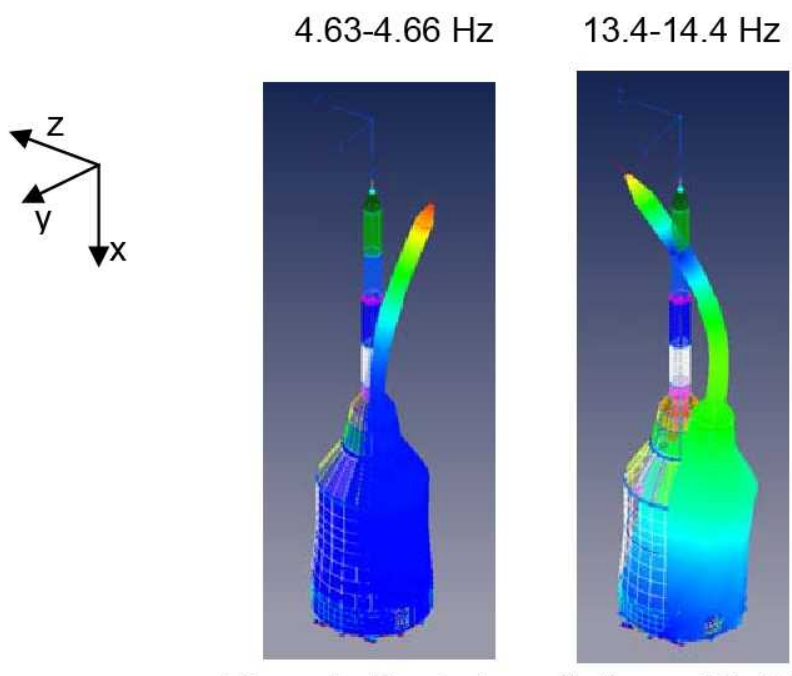

\section{2-26.3 Hz}

Figure 5 Pre-test predictions of first three pairs of bending modes
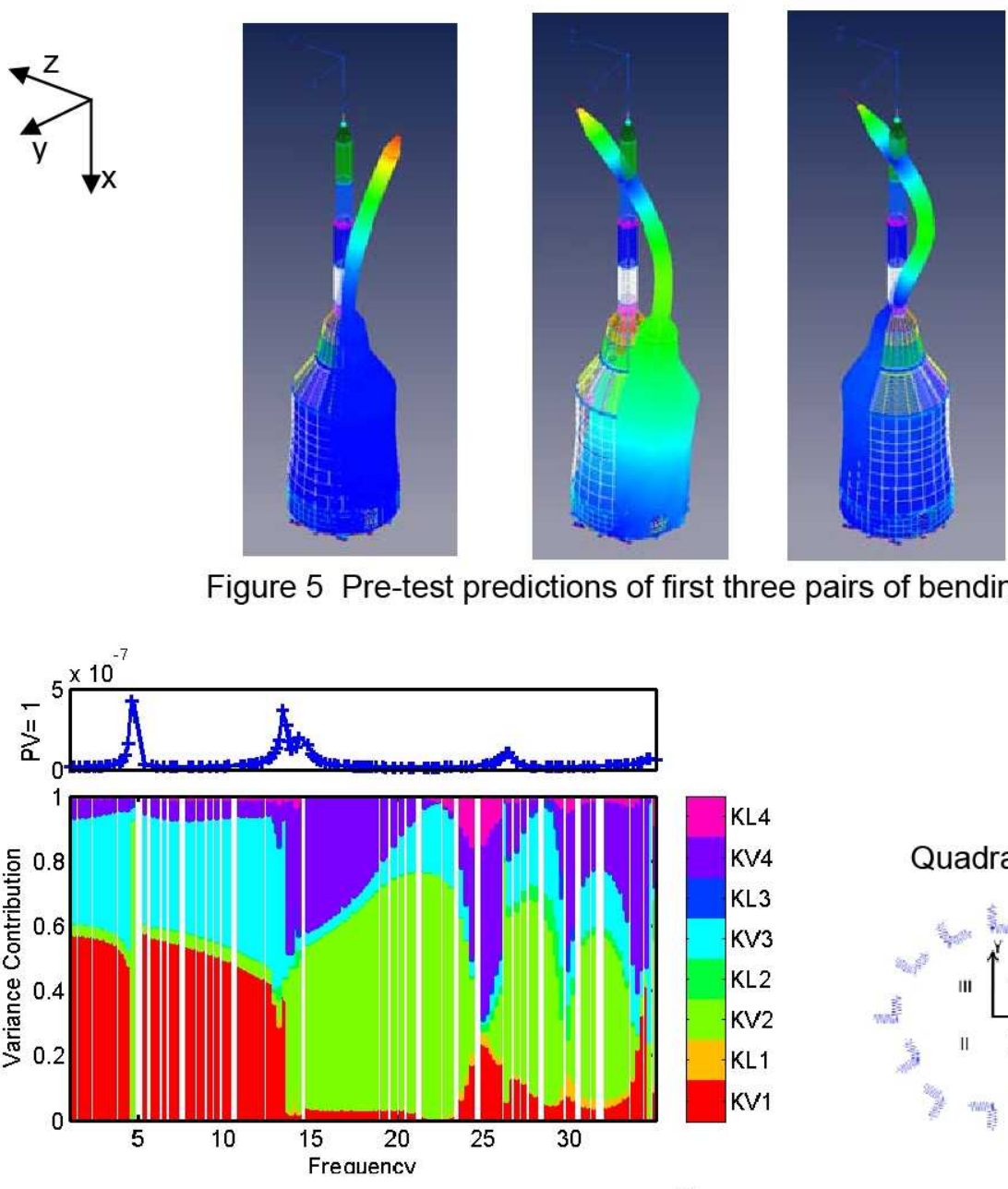

Figure 5 Pre-test predictions of first three pairs of bending modes

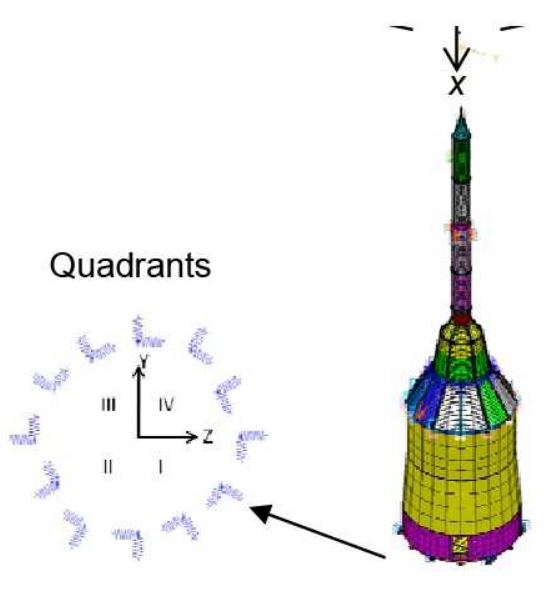

Figure 6 Parameter contributions to variance of the $1^{\text {st }}$ principal value due to longitudinal and vertical boundary stiffness variations. 


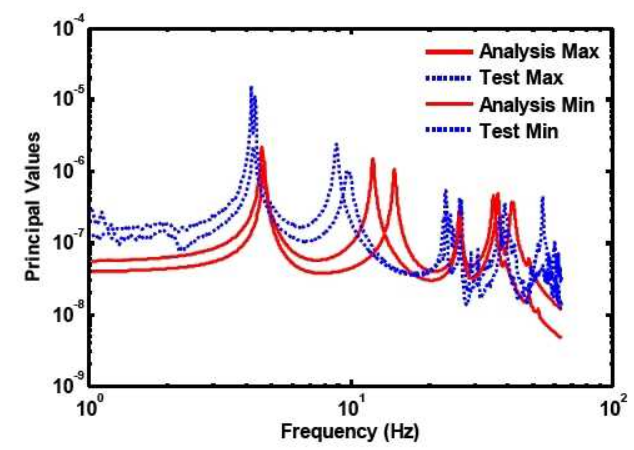

a) pre-test model bounds

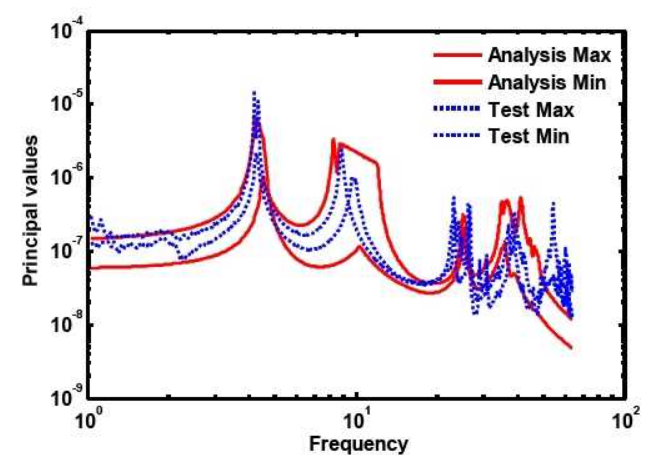

b) Updated bounds

Figure 7 Comparison of SS5 principal value bounds for a) pre-test model and b) after updates to parameter ranges

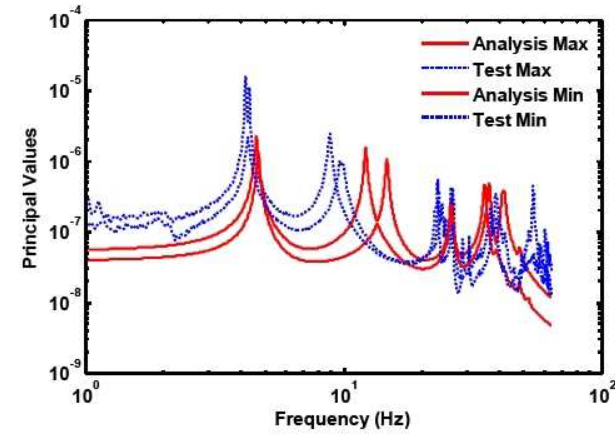

a) pre-test nominal model

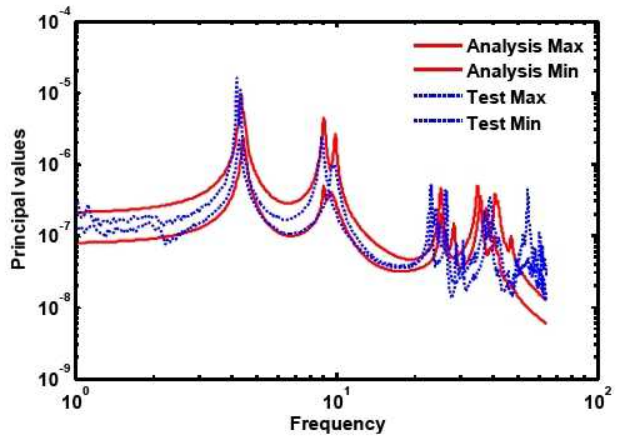

b) optimized

Figure 8 Comparison of SS5 principal values for a) pre-test model b) model with optimized boundary parameters

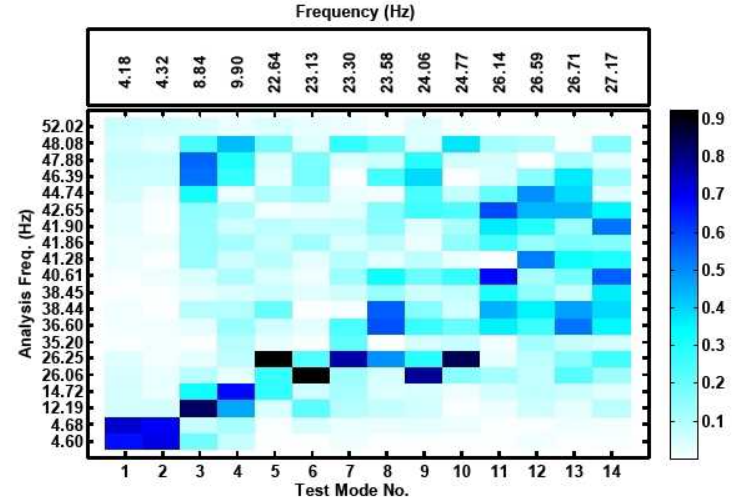

a) pre-test model

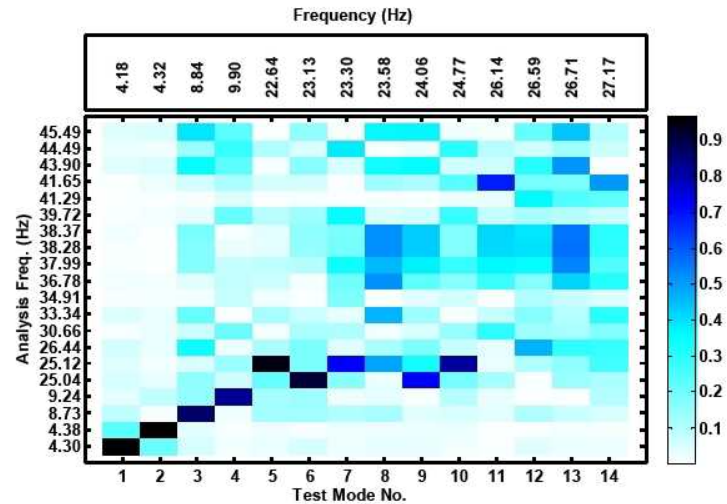

b) optimized

Figure 9 SS5 orthogonality results with a) pre-test model and b) with optimized boundary parameters 


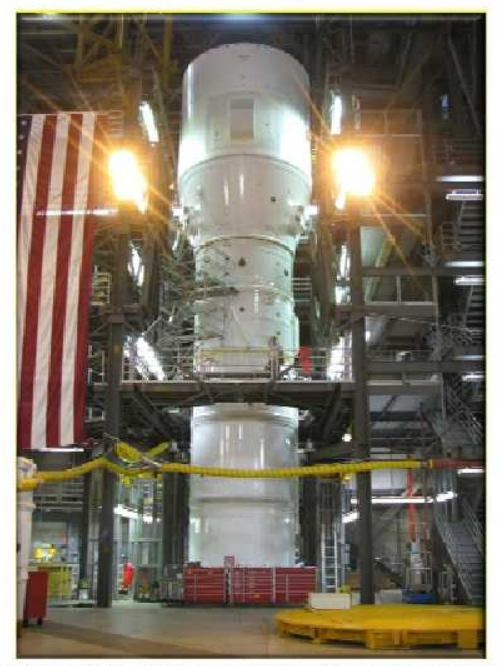

Figure 10 Ares I-X SS1 test article

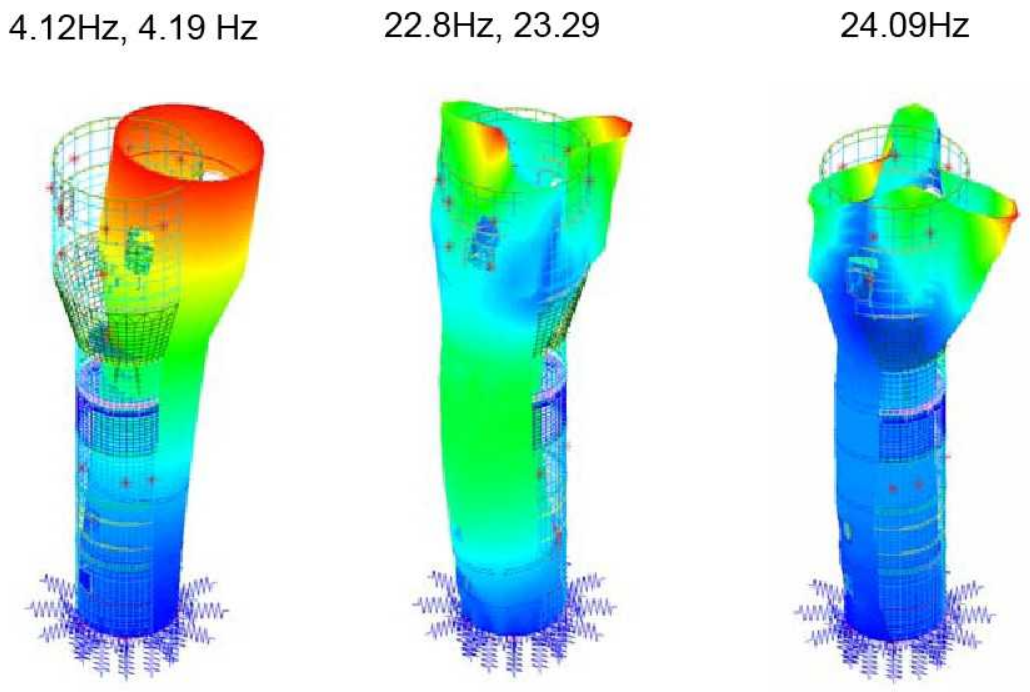

Figure 11 SS1 pre-test predictions
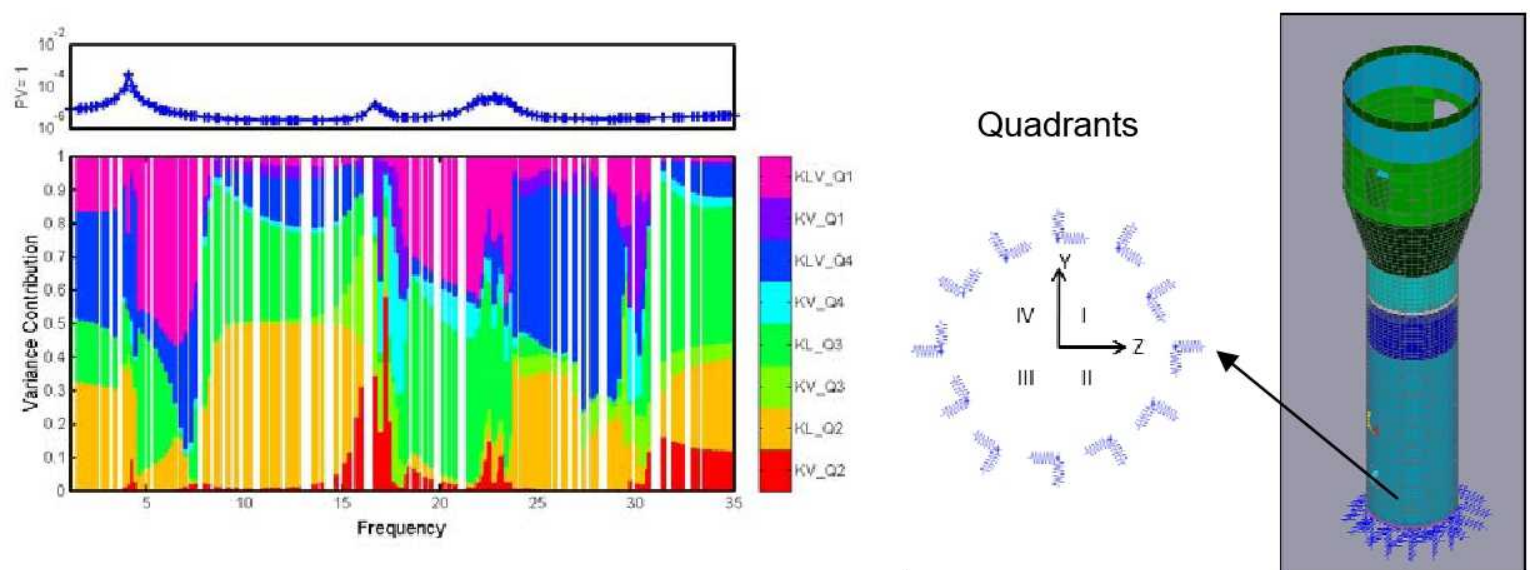

Figure 12 SS1 parameter contributions to variance of the $1^{\text {st }}$ principal value due to boundary stiffness variations 


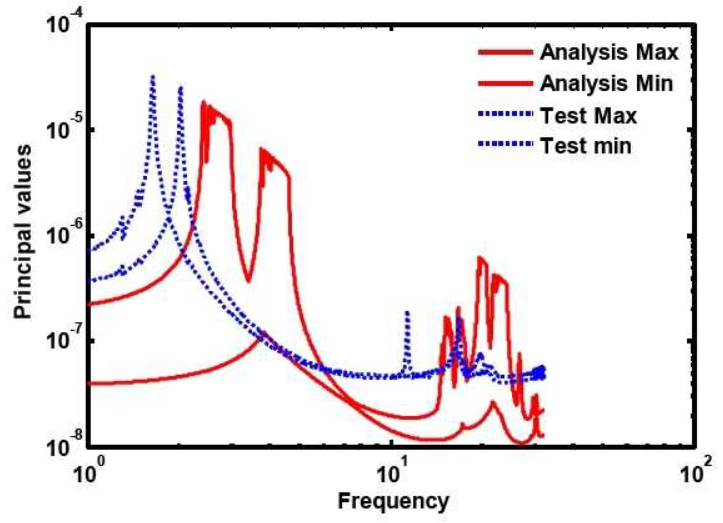

a) Pre-test model bounds

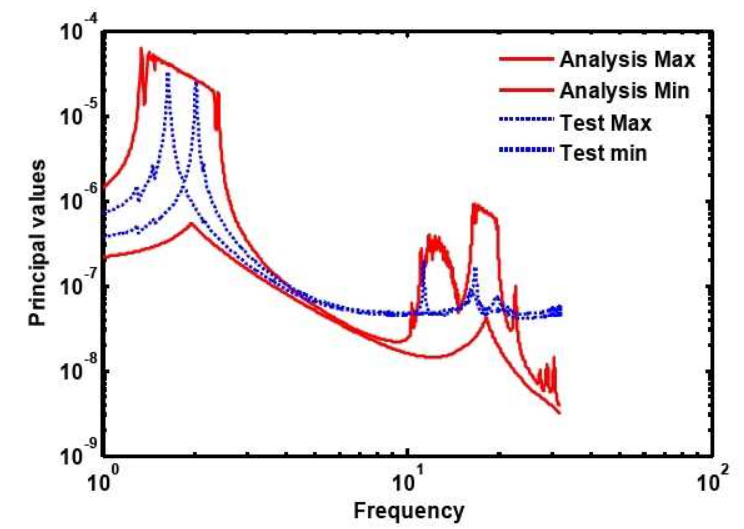

b) updated bounds

Figure 13 Comparison of SS1 PV bounds for a) pre-test model and b) updated boundary stiffnes bounds

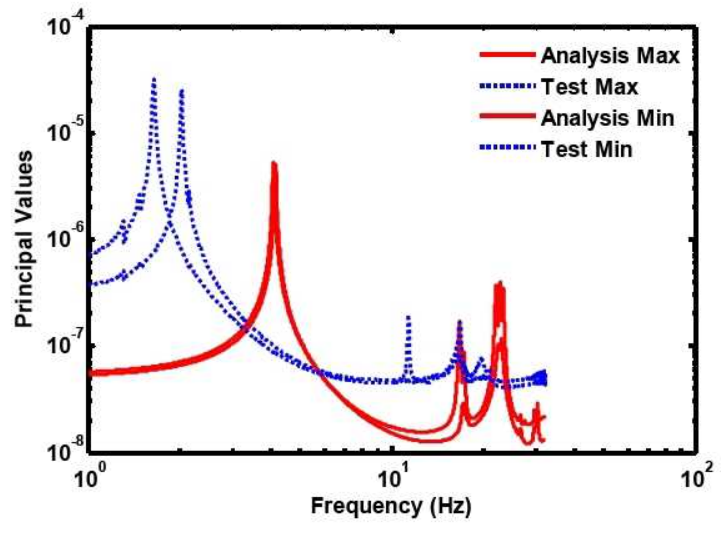

a) Pre-test model

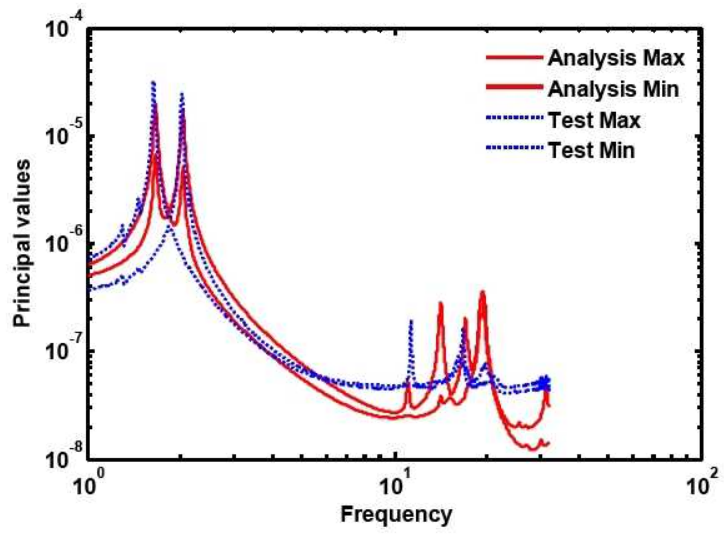

b) optimized boundary

Figure 14 Comparison of SS1 PV with a) pre-test model and b) optimized boundary stiffness model

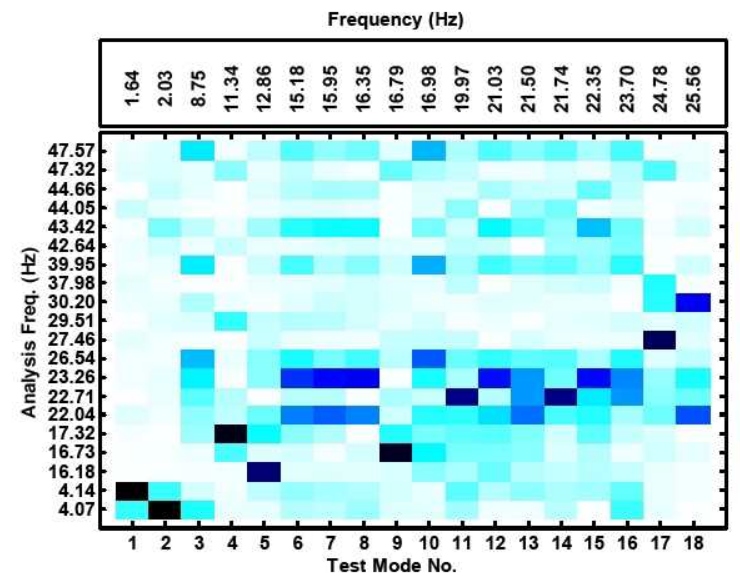

a) Pre-test model

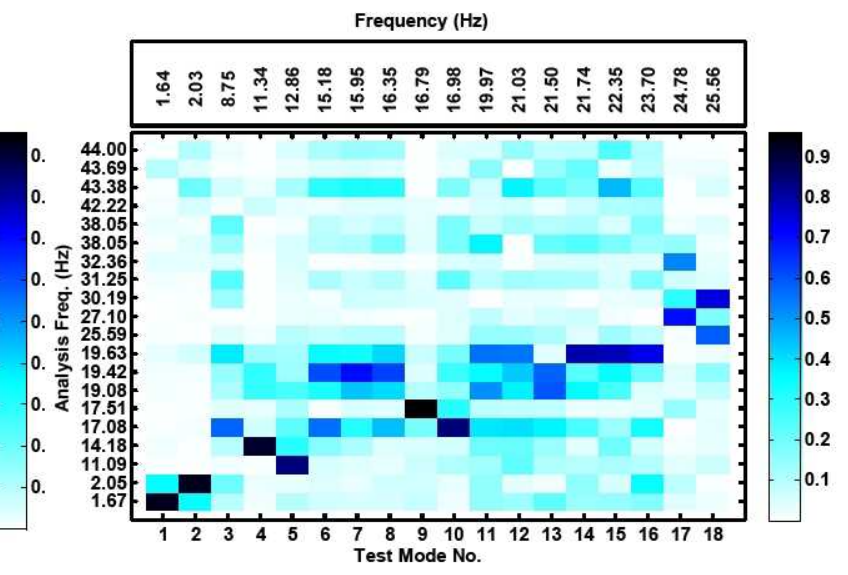

b) updated

Figure 15 SS1 orthogonality results with pre-test model and with updated boundary stiffness model 


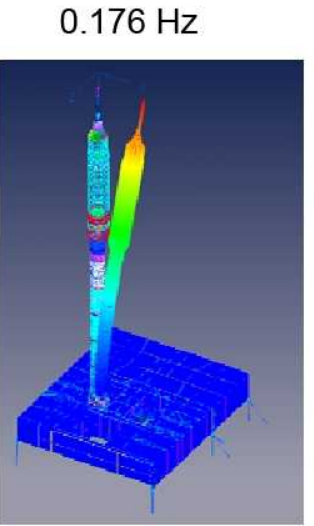

$3.248 \mathrm{~Hz}$
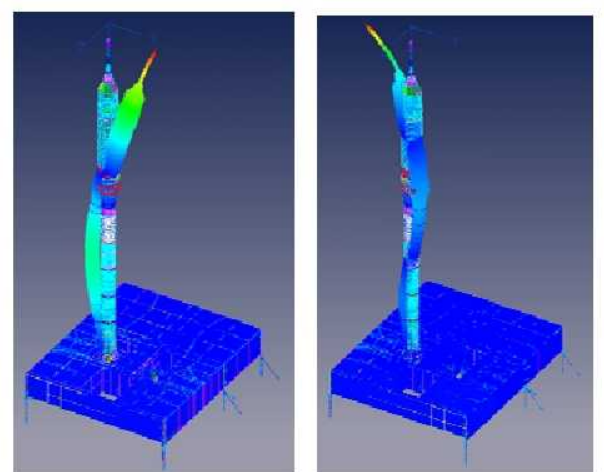

Figure 16 Flight vehicle target modes
$4.78 \mathrm{~Hz}$
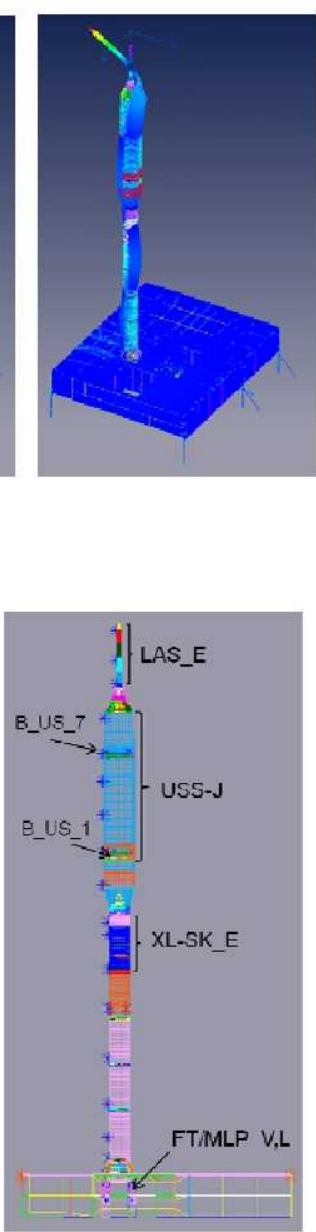

Figure 17 Parameter contributions to variance of the $1^{\text {st }} \mathrm{PV}$ for the flight vehicle

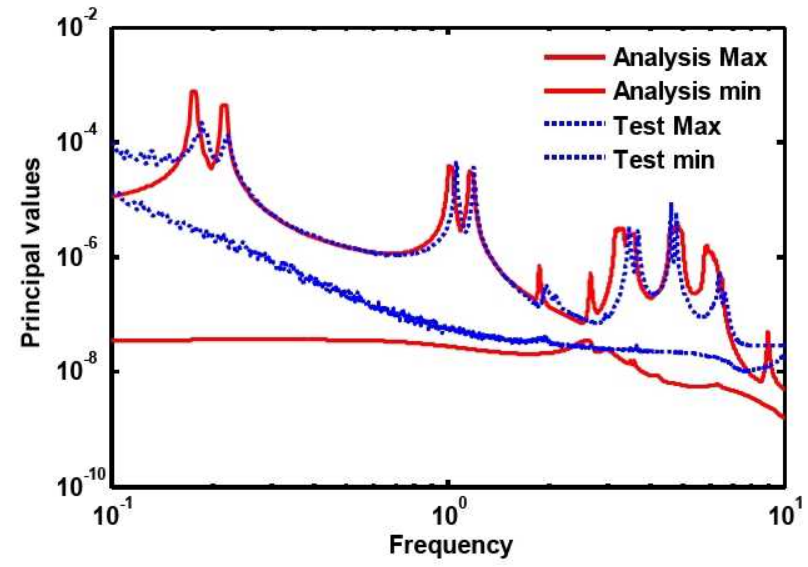

Figure 18 Comparison of flight vehicle PV bounds for pre-test model and test (assumed damping for model is $0.5 \%$ ) 


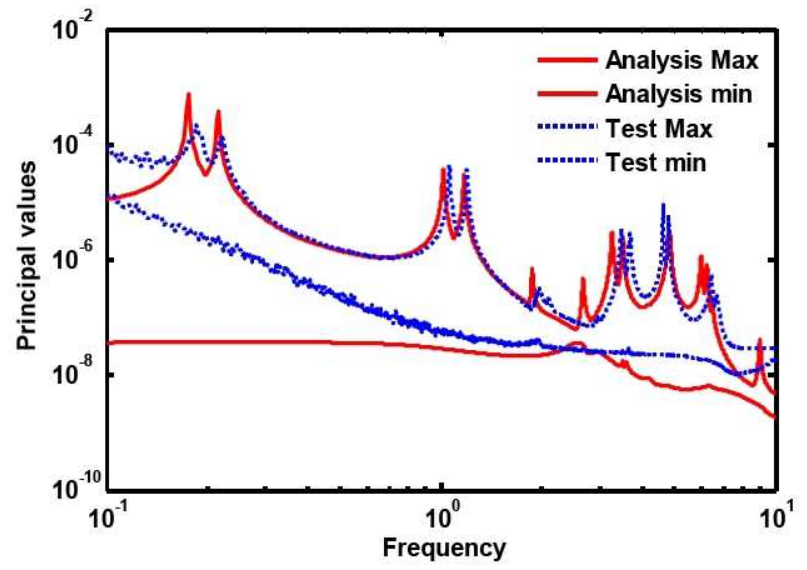

Figure 19 Comparison of flight vehicle PV for pre-test model and test

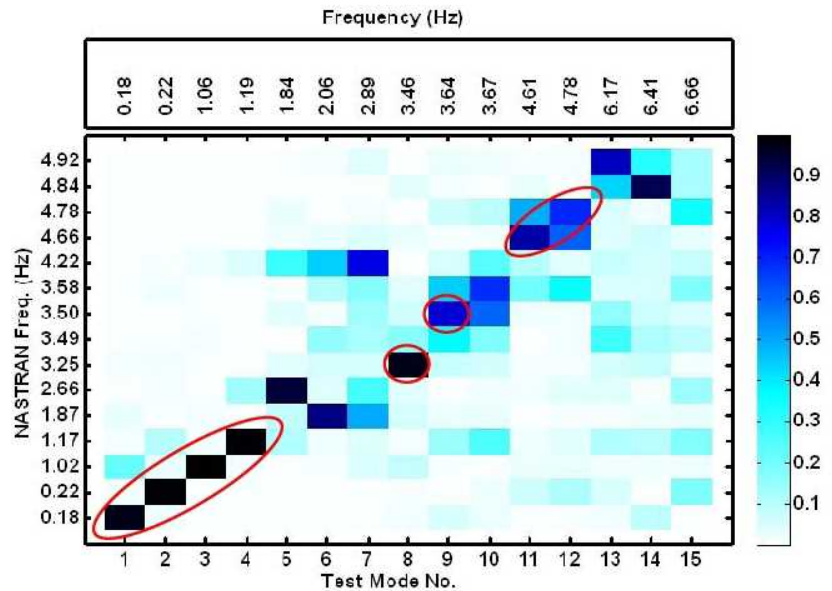

Figure 20 Flight test vehicle orthogonality results with pre-test and updated model 